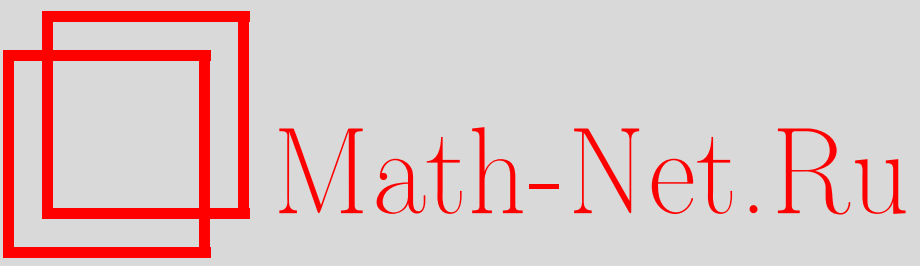

П. А. Вельмисов, Ю. А. Тамарова, Е. П. Семенова, Асимптотические уравнения газовой динамики: качественный анализ, построение решений, приложения, Итоги науки и техн. Сер. Соврем. мат. и ее прил. Темат. обз., 2019, том $165,47-62$

DOI: https://doi.org/10.36535/0233-6723-2019-165-47-62

Использование Общероссийского математического портала Math-Net.Ru подразумевает, что вы прочитали и согласны с пользовательским соглашением

http://www.mathnet.ru/rus/agreement

Параметры загрузки:

IP: 54.80 .73 .141

26 апреля 2023 г., 11:33:41 


\title{
АСИМПТОТИЧЕСКИЕ УРАВНЕНИЯ ГАЗОВОЙ ДИНАМИКИ: КАЧЕСТВЕННЫЙ АНАЛИЗ, ПОСТРОЕНИЕ РЕШЕНИЙ, ПРИЛОЖЕНИЯ
}

\author{
(C) 2019 г. П. А. ВЕЛЬМИСОВ, Ю. А. ТАМАРОВА, Е. П. СЕМЕНОВА
}

\begin{abstract}
АннотАция. Предложены асимптотические разложения для потенциала скорости, на основе которых выводятся асимптотические уравнения газовой динамики для безвихревых изоэнтропических течений идеального газа: уравнение линейной теории, нелинейное уравнение для сверхзвуковых течений, нелинейное трансзвуковое уравнение. Построены некоторые точные частные решения асимптотического нелинейного трансзвукового уравнения, учитывающего поперечные по отношению к основному потоку возмущения. На основе линейного асимптотического уравнения исследуется динамическая устойчивость упругого деформируемого элемента канала при дозвуковой скорости потока газа или жидкости. Исследование устойчивости проводится в постановке, соответствующей малым возмущениям однородного потока и малым деформациям упругого элемента, и основано на построении положительно определенного функционала, при этом получены достаточные условия устойчивости.
\end{abstract}

Ключевъе слова: аэродинамика, дифференциальное уравнение с частными производными, асимптотическое разложение, трансзвуковое течение газа, канал, сопло Лаваля, аэрогидроупругость, динамическая устойчивость.

\section{ASYMPTOTIC EQUATIONS OF GAS DYNAMICS: QUALITATIVE ANALYSIS, CONSTRUCTION OF SOLUTIONS, AND APPLICATIONS}

\author{
(c) 2019 P. A. VELMiSOV, YU. A. TAMAROVA, E. P. SEMENOVA
}

\begin{abstract}
In this paper, we propose asymptotic expansions for the velocity potential and obtain asymptotic equations of gas dynamics for irrotational isentropic flows of an ideal gas: an equation of linear theory, a nonlinear equation for supersonic flows, and a nonlinear transonic equation. We construct some exact particular solutions for the asymptotic nonlinear transonic equation, which takes into account transverse perturbations. Based on a linear asymptotic equation, we examine the dynamic stability of an elastic deformable element of a channel at a subsonic flow rate of a gas or liquid. The study of stability is carried out in a statement corresponding to small perturbations of a homogeneous flow and small deformations of an elastic element, and is based on the construction of a positive definite functional. Sufficient stability conditions are obtained.
\end{abstract}

Keywords and phrases: aerodynamics, partial differential equation, asymptotic expansion, transonic gas flow, channel, de Laval nozzle, aerohydroelasticity, dynamic stability.

AMS Subject Classification: 35C20, 76G25

Работа выполнена при поддержке Российского фонда фундаментальных исследований (проект № 18-41-730015). 


\section{СОДЕРЖАНИЕ}

1. Введение . . . . . . . . . . . . . . . . . . . . . . . . . . 48

2. Асимптотические уравнения газовой динамики: вывод уравнений и условий . . . . . . 49

3. Некоторые частные решения трансзвуковых уравнений . . . . . . . . . . . . . . . . . 53

4. Исследование динамической устойчивости упругого элемента стенки канала . . . . . . 56

Список литературы . . . . . . . . . . . . . . . . . . . . . . . . . . . . . . . . . 59

\section{1. ВВЕДЕНИЕ}

Задачи аэродинамики, связанные с переходом через скорость звука, имеют большое прикладное значение. Одной из важных задач является задача обтекания тела безграничным потоком. Этот класс задач играет значительную роль в аэродинамике крыла и снаряда, так как полет многих современных летательных аппаратов происходит в условиях трансзвукового режима обтекания. Результаты анализа и расчета задач обтекания используются при решении ряда актуальных проблем высокоскоростной (реактивной) авиации и внешней баллистики. Другим важным направлением применения теории трансзвуковых течений является расчет движения газа в соплах реактивных двигателей.

Трансзвуковые течения характерны тем, что в них одновременно присутствуют сверхзвуковая область (область гиперболичности) и дозвуковая (область эллиптичности), при этом граница раздела (поверхность параболичности), которая является звуковой поверхностью, заранее не известна. Смешанный тип и нелинейность уравнений, описывающих движение газа с околозвуковой скоростью, делают проблему расчета трансзвуковых течений чрезвычайно сложной. Аналитическое исследование трансзвуковых течений на основе точных уравнений газовой динамики фактически невозможно, поэтому для изучения движения газа в околозвуковом диапазоне скоростей применяется приближенное (асимптотическое) уравнение.

Основы теории трансзвуковых течений были заложены на заре развития сверхзвуковой авиации в 1946-1950 гг. С. В. Фальковичем [47], Т. Карманом [53], Л. В. Овсянниковым [41], Ф. И. Франклем [48], К. Г. Гудерлеем [30] и др. Для нестационарных течений приближенное околозвуковое уравнение было получено в [54]; при этом основная сложность исследования заключается в том, что даже в приближенной постановке указанная проблема нелинейна. Дальнейшее развитие теория трансзвуковых течений получила в работах О. С. Рыжова [36-38,42-44], Ю. Б. Лифшица [36-38], Э. Г. Шифрина [49]. Исследованию нестационарных околозвуковых течений посвящены работы Т. С. Adamson [50], П. А. Вельмисова [15,27], С. В. Фальковича [27]. В работах S. Tomotika [58,59], K. Tamada [58], П. А. Вельмисова [15,16,27], О. С. Рыжова [36,37,43,44], Ю. Б. Лифшица $[36,37]$, С. В. Фальковича $[27,47]$ изучаются течения в соплах Лаваля. Примеры расчетов в соплах и при обтекании летательных аппаратов для установившихся и неустановившихся течений представлены в $[15,16,21,27,43,47,50]$. На структуру течений газа в некоторых случаях существенное влияние оказывают вязкость и теплопроводность. Учет вязкости проводился в работах M. Sichel [56,57], О. С. Рыжова, Г. М. Шефтера [45], П. А. Вельмисова, С. В. Фальковича [28]. Вследствие нелинейности трансзвукового уравнения большой интерес представляет вопрос отыскания точных частных решений, которые необходимы для того, чтобы на основе примеров течений, построенных с их помощью, изучить характерные свойства околозвуковых течений. Эти решения также нужны для проверки различных численных методов исследования трансзвуковых течений, в этом случае они являются тестирующими примерами. Проблеме построения частных решений посвящены работы перечисленных выше авторов, а также авторов настоящей статьи $[18,24-26,32,34,51,52]$.

При проектировании и эксплуатации конструкций, приборов, устройств различного назначения, взаимодействующих с потоком газа, важной проблемой является обеспечение надежности их функционирования и увеличение сроков службы. Подобные проблемы присущи многим отраслям техники. В частности, такого рода задачи возникают в авиаракетостроении, при проектировании антенных установок, высоких наземных сооружений и т. д. Существенное значение при расчете 
конструкций, взаимодействующих с потоком газа, имеет исследование устойчивости деформируемых элементов, так как воздействие потока может приводить к ее потере. В качестве примеров потери динамической устойчивости можно указать: флаттер крыла самолета, панельный флаттер пластин и оболочек, обтекаемых потоком, например флаттер панели обшивки самолета или ракеты; срывной флаттер лопаток турбин и винтов; колебания проводов, дымовых труб, висячих мостов и т. д. Устойчивости упругих тел, взаимодействующих с потоком газа, посвящено большое количество проведенных в последние десятилетия теоретических и экспериментальных исследований. Они представлены в работах С. М. Белоцерковского, Б. К. Скрипача, В. Г. Табачникова, Э. И. Григолюка, В. В. Болотина, А. С. Вольмира, Р. Е. Лампера, Л. Г. Шандарова, Ю. Н. Новичкова, Р. Л. Бисплингхоффа, Х. Эшли, Р. Л. Халфмана, Я. Ц. Фына, Г. Фершинга, Е. Х. Доуэлла, А. Г. Горшкова, А. А. Ильюшина, И. А. Кийко, С. Д. Алгазина, А. А. Мовчана, Дж. Майлса, Я. Г. Пановко, И. И. Губанова, М. А. Ильгамова, Б. Ю. Кудрявцева, Д. М. Минасяна, В. И. Морозова, В. В. Овчинникова, Л. И. Могилевича, П. А. Вельмисова и др. Среди последних исследований по динамике и устойчивости трубопроводов и их элементов, при протекании внутри них потока жидкости или газа, следует отметить исследования Л. И. Могилевича, А. А. Поповой, В. В. Мокеева, Б. А. Ершова, Ю. П. Барметова, И. А. Дободейча, А. В. Звягина, В. Г. Соколова, А. В. Березнева, М. P. Paidoussis и многих других отечественных и зарубежных ученых (см., например, $[14,31,33,39,40,46,55])$. Среди работ авторов данной статьи по исследованию динамики и устойчивости упругих тел, взаимодействующих с потоком газа, отметим $[1-13,19,20,22,23,29]$.

В данной статье получены уравнения, являющиеся обобщением классических уравнений в случае учета поперечных воздействий:

1) линейное уравнение, которое применяется для описания как дозвуковых, так и сверхзвуковых течений;

2) нелинейное уравнение, которое описывает сверхзвуковое течение в окрестности ударной волны;

3) нелинейное уравнение, описывающее трансзвуковые течения газа.

Выведены асимптотические условия на фронте ударной волны и условия на обтекаемой поверхности, а также записаны уравнения звуковой поверхности и асимптотические формулы для определения давления. Построены некоторые точные частные решения как для классического трансзвукового уравнения, так и для полученного в статье нелинейного трансзвукового уравнения, учитывающего поперечные возмущения. Указаны их приложения к решению ряда задач трансзвуковой аэродинамики.

В качестве приложения асимптотического линейного уравнения рассматривается задача исследования динамической устойчивости упругого элемента стенки канала. Модель описывается связанной системой дифференциальных уравнений в частных производных для двух неизвестных функций - потенциала скорости газа или жидкости и деформации упругого элемента. Задача исследуется в линейной постановке, соответствующей малым возмущениям потока в канале и малым деформациям упругого элемента. На основе построения смешанного функционала получены достаточные условия устойчивости, налагающие ограничения на параметры механической системы.

\section{2. АСИМПТОТИЧЕСКИЕ УРАВНЕНИЯ ГАЗОВОЙ ДИНАМИКИ: ВЫВОД УРАВНЕНИЙ И УСЛОВИЙ}

2.1. Линейные асимптотические уравнения. Безвихревые изэнтропические течения газа в декартовых координатах $x, y, z$ описываются уравнением

$$
\begin{gathered}
\Phi_{t t}+2 \Phi_{x} \Phi_{x t}+2 \Phi_{y} \Phi_{y t}+2 \Phi_{z} \Phi_{z t}+2 \Phi_{x} \Phi_{z} \Phi_{x z}+2 \Phi_{x} \Phi_{y} \Phi_{x y}+2 \Phi_{z} \Phi_{y} \Phi_{z y}+\Phi_{x}^{2} \Phi_{x x}+\Phi_{y}^{2} \Phi_{y y}+ \\
+\Phi_{z}^{2} \Phi_{z z}-\left(\Phi_{x x}+\Phi_{y y}+\Phi_{z z}\right)\left(a_{0}^{2}+\frac{\chi-1}{2} V_{0}^{2}-(\chi-1)\left(\Phi_{t}+\frac{1}{2} \Phi_{x}^{2}+\frac{1}{2} \Phi_{y}^{2}+\frac{1}{2} \Phi_{z}^{2}\right)\right)=0 \\
\left(\frac{a}{a_{0}}\right)^{2}=\left(\frac{\rho}{\rho_{0}}\right)^{2}=\left(\frac{P}{P_{0}}\right)^{2}=1+\frac{\chi-1}{2} \frac{V_{0}^{2}}{a_{0}^{2}}-\frac{\chi-1}{2 a_{0}^{2}}\left(2 \Phi_{t}+\Phi_{x}^{2}+\Phi_{y}^{2}+\Phi_{z}^{2}\right) .
\end{gathered}
$$


Здесь $\Phi(x, y, z, t)$ - потенциал скорости, $x, y, z$ - декартовы координаты, $t$ - время, $a$ - скорость звука, $\rho$ - плотность, $P$ - давление, $\chi$ - показатель адиабаты Пуассона; $V_{0}$ - скорость однородного потока, $a_{0}, \rho_{0}, P_{0}$ - значения скорости звука, плотности и давления в однородном потоке; индексы снизу обозначают частные производные.

Предполагая, что имеют место малые возмущения однородного потока, движущегося со скоростью $V_{0}$ в направлении оси $O x$, представим $\Phi(x, y, z, t)$ в виде

$$
\Phi(x, y, z, t)=V_{0} x+\psi(y, z, t)+\varepsilon \varphi(x, y, z, t)+\ldots, \quad \varepsilon \ll 1 .
$$

В $(2.3)$ функция $\varphi(x, y, z, t)$ определяет основное течение, а функция $\psi(y, z, t)$ задает поперечное возмущение. Тогда, подставляя $(2.3)$ в $(2.1)$ и оставляя члены порядка единицы и $\varepsilon$, получим нелинейное уравнение для функции $\psi(y, z, t)$ и линейное уравнение для функции $\varphi(x, y, z, t)$, которые несложно записываются, но здесь не приводятся вследствие их громоздкости.

При $\psi=0$ уравнение линейной теории для $\varphi(x, y, z, t)$ имеет вид

$$
\varphi_{t t}+2 V_{0} \varphi_{x t}+V_{0}^{2} \varphi_{x x}-a_{0}^{2}\left(\varphi_{x x}+\varphi_{y y}+\varphi_{z z}\right)=0 .
$$

В стационарном плоском случае получим уравнение

$$
\left(M_{0}^{2}-1\right) \varphi_{x x}-\varphi_{y y}=0, \quad M_{0}=\frac{V_{0}}{a_{0}},
$$

которое при $M_{0}>1$-волновое уравнение гиперболического типа, а при $M_{0}<1$-уравнение эллиптического типа, которое приводится к уравнению Лапласа. Давление определяется по формуле

$$
P=P_{0}-\rho_{0} \varepsilon\left(\varphi_{t}+V_{0} \varphi_{x}\right) .
$$

Выведем условие на обтекаемой поверхности, задав ее в виде

$$
f(x, y, z, t)=f_{0}(y, z)+\varepsilon f_{1}(x, y, z, t)+\cdots=0 .
$$

Подставляя (2.3) и (2.5) в точное условие непротекания

$$
\Phi_{x} f_{x}+\Phi_{y} f_{y}+\Phi_{z} f_{z}=-f_{t}
$$

и оставляя старшие члены, получим

$$
V_{0} f_{1 x}+\varphi_{y} f_{0 y}+\varphi_{z} f_{0 z}=-f_{1 t} .
$$

В плоском случае $f(x, y, t)=y-y_{0}-\varepsilon w(x, t)\left(y=y_{0}+\varepsilon w(x, t)\right)$, и условие непротекания примет вид

$$
\varphi_{y}\left(x, y_{0}, t\right)=w_{t}+V_{0} w_{x} .
$$

Уравнение характеристик для (2.4) имеет вид

$$
\left(\frac{\partial x}{\partial t}\right)^{2}-2 V_{0} \frac{\partial x}{\partial t}+V_{0}^{2}-a_{0}^{2}\left(1+\left(\frac{\partial x}{\partial y}\right)^{2}+\left(\frac{\partial x}{\partial z}\right)^{2}\right)=0 .
$$

2.2. Вывод асипмтотических уравнений и условий для сверхзвуковых течений с поперечным возмущением. Введем асимптотическое разложение

$$
\Phi=V_{0} x+\varepsilon \psi(y, z, t)+\varepsilon^{2} \varphi\left(\xi^{0}, y, z, t\right)+\ldots, \quad \xi^{0}=\frac{\xi}{\varepsilon}, \quad \xi=x-\beta y, \quad \beta=\sqrt{M_{0}^{2}-1},
$$

где $\varepsilon$-малый параметр, $M_{0}=V_{0} / a_{0}$ - число Маха. Подставляя (2.6) в (2.1), получим в первом приближении для функции $\varphi\left(\xi^{0}, y, z, t\right)$ уравнение

$$
2 V_{0} \varphi_{\xi^{0} t}+2 \beta a_{0}^{2} \varphi_{\xi^{0} y}+\left[(\chi+1) V_{0} M_{0}^{2} \varphi_{\xi^{0}}+(\chi-1) M_{0}^{2} \psi_{t}-2 V_{0} \beta \psi_{y}\right] \varphi_{\xi^{0} \xi^{0}}=a_{0}^{2}\left(\psi_{y y}+\psi_{z z}\right)-\psi_{t t} .
$$

В $(2.7)$ функция $\psi(y, z, t)$, являющаяся произвольной, позволяет учесть, например, боковое аэродинамическое воздействие на обтекаемое тело. Уравнение (2.7) описывает течение в окрестности ударной волны, мало отличающейся от характеристики $\xi=$ const. В [17] разложение (2.6) используется для исправления линейной теории в окрестности головной ударной волны при обтекании профиля сверхзвуковым потоком газа. 
Подставляя (2.6) в точное условие непротекания и оставляя члены старшего порядка,

$$
-V_{0} y_{1 \xi^{0}}=y_{0 t}, \quad \psi_{y}+\psi_{z} y_{0 z}=\left(\beta+y_{1 \xi^{0}}\right) \varphi_{\xi^{0}}+V_{0} y_{2 \xi^{0}}+y_{1 t},
$$

получим условие на обтекаемой поверхности:

$$
y=y_{0}(z, t)+y_{1}\left(\xi^{0}, z, t\right) \varepsilon+y_{2}\left(\xi^{0}, z, t\right) \varepsilon^{2}+\ldots
$$

Значения $\psi_{y}, \psi_{z}, \varphi_{\xi^{0}}$ вычисляются при $y=y_{0}(z, t)$. Условия на фронте ударной волны $\xi^{0}=$ $\xi^{0}(y, z, t)$ получим из условий Ренкина-Гюгонио, подставляя в них разложение (2.6) и оставляя члены старшего порядка:

$$
\frac{\chi+1}{2} M_{0}^{2} V_{0}\left(\varphi_{\xi^{0}}+\varphi_{\xi^{0}}^{*}\right)=V_{0} \frac{\partial \xi^{0}}{\partial t}+\beta a_{0}^{2} \frac{\partial \xi^{0}}{\partial y}+V_{0} \beta \psi_{y}-\frac{(\chi-1) M_{0}^{2}}{2} \psi_{t}, \quad \varphi=\varphi^{*} .
$$

В (2.8) функции $\varphi$ и $\varphi^{*}$ соответствуют течению с разных сторон от ударной волны. Если в $(2.8)$ положить $\varphi \equiv \varphi^{*}$, то получим характеристическое уравнение для (2.7). Подставляя (2.6) в выражение для давления (2.2), проводя разложение в ряд Тейлора и оставляя старшие по порядку члены, получим асимптотическую формулу для определения давления:

$$
P=P_{0}\left(1-\frac{\chi}{a_{0}^{2}} \varepsilon\left(\psi_{t^{0}}+V_{0} \varphi_{\xi^{0}}\right)\right) .
$$

2.3. Вывод асимптотических уравнений и условий для трансзвуковых течений с поперечным возмущением. В цилиндрических безразмерных координатах $x, r, \theta$ уравнения (2.1), (2.2) примут вид

$$
\begin{gathered}
\Phi_{t t}+2 \Phi_{x} \Phi_{x t}+2 \Phi_{r} \Phi_{r t}+\frac{2}{r^{2}} \Phi_{\theta} \Phi_{\theta t}+2 \Phi_{x} \Phi_{r} \Phi_{r x}+\frac{2}{r^{2}} \Phi_{\theta} \Phi_{r} \Phi_{\theta r}+\frac{2}{r^{2}} \Phi_{x} \Phi_{\theta} \Phi_{\theta x}+\Phi_{x}^{2} \Phi_{x x}+ \\
+\Phi_{r}^{2} \Phi_{r r}+\frac{1}{r^{4}} \Phi_{\theta}^{2} \Phi_{\theta \theta}-a^{2}\left(\Phi_{x x}+\Phi_{r r}+\frac{1}{r} \Phi_{r}+\frac{1}{r^{2}} \Phi_{\theta \theta}\right)=0 \\
a^{2}(\Phi)=\rho^{\chi-1}=P^{\frac{\chi-1}{\chi}}=\frac{\chi+1}{2}-\frac{\chi-1}{2}\left(2 \Phi_{t}+\Phi_{x}^{2}+\Phi_{r}^{2}+\frac{1}{2 r^{2}} \Phi_{\theta}^{2}\right) .
\end{gathered}
$$

Введем для $\Phi(x, r, \theta, t)$ асимптотическое разложение

$$
\Phi=x+\varepsilon \psi\left(r, \theta, t^{0}\right)+\varepsilon^{3} \varphi\left(x^{0}, r, \theta, t^{0}\right)+\ldots, \quad x=\varepsilon x^{0}, \quad t=\frac{1}{\varepsilon} t^{0},
$$

где $\varepsilon$ - малый параметр, функция $\varphi\left(x^{0}, r, \theta, t^{0}\right)$ определяет основное течение, а функция $\psi\left(r, \theta, t^{0}\right)$ задает поперечное возмущение. Подставляя $(2.11)$ в $(2.9)-(2.10)$ и оставляя члены старшего порядка, получим для функции $\varphi\left(x^{0}, r, \theta, t^{0}\right)$ трансзвуковое уравнение:

$$
2 \varphi_{x^{0} t^{0}}+(\chi+1) \varphi_{x^{0}} \varphi_{x^{0} x^{0}}+2 \psi_{r} \varphi_{x^{0} r}+\frac{2}{r^{2}} \psi_{\theta} \varphi_{x^{0} \theta}-\Delta \varphi+\frac{\chi-1}{2}\left(2 \psi_{t^{0}}+\psi_{r}^{2}+\frac{1}{r^{2}} \psi_{\theta}^{2}\right) \varphi_{x^{0} x^{0}}=L(\psi) .
$$

В 2.12 введены обозначения

$$
\begin{gathered}
-L(\psi) \equiv \psi_{t^{0} t^{0}}+2 \psi_{r} \psi_{r t^{0}}+\frac{2}{r^{2}} \psi_{\theta} \psi_{\theta t^{0}}+\psi_{r}^{2} \psi_{r r}+\frac{1}{r^{4}} \psi_{\theta}^{2} \psi_{\theta \theta}+\frac{2}{r^{2}} \psi_{\theta} \psi_{r} \psi_{r \theta}-\frac{1}{r^{3}} \psi_{r} \psi_{\theta}^{2}, \\
\Delta \varphi \equiv \varphi_{r r}+\frac{1}{r} \varphi_{r}+\frac{1}{r^{2}} \varphi_{\theta \theta} .
\end{gathered}
$$

Функция $\psi\left(r, \theta, t^{0}\right)$ удовлетворяет уравнению Лапласа $\Delta \psi=0$. Если $\psi \equiv 0$, то получим классическое трансзвуковое уравнение Линя-Рейсснера-Тзяна в цилиндрических координатах:

$$
2 \varphi_{x^{0} t^{0}}+(\chi+1) \varphi_{x^{0}} \varphi_{x^{0} x^{0}}-\varphi_{r r}-\frac{1}{r} \varphi_{r}-\frac{1}{r^{2}} \varphi_{\theta \theta}=0,
$$

которое в стационарном случае переходит в уравнение смешанного типа Кармана-Фальковича:

$$
(\chi+1) \varphi_{x^{0}} \varphi_{x^{0} x^{0}}-\varphi_{r r}-\frac{1}{r} \varphi_{r}-\frac{1}{r^{2}} \varphi_{\theta \theta}=0 .
$$

В плоском случае (на плоскости годографа) это уравнение с помощью преобразования Лежандра приводится к линейному уравнению Трикоми. 
Уравнение 2.12 описывает трансзвуковые течения газа, возникающие при воздействии на обтекаемое тело бокового (по отношению к основному направлению движения, совпадающему с направлением оси $x$ ) возмущения основного трансзвукового потока (для возмущающего поперечного течения $-\Phi_{r}, \Phi_{\theta} \sim \varepsilon$, для основного течения $\left.-\Phi_{r}, \Phi_{\theta} \sim \varepsilon^{3}\right)$. Для внутреннего обтекания, например, для течений в соплах, таким возмущением может быть закрутка потока $(\psi=\Gamma(t) \theta)$. Для внешнего обтекания летательных аппаратов таким возмущением является, например, боковой ветер $\psi=V_{\infty}(t) r \cos (\theta+\alpha(t))$, меняющий свою скорость с течением времени.

В случае $\psi=V_{\infty} \cos \theta\left(r+R^{2} / r\right)$ получим асимптотическое уравнение, описывающее течения, возникающие при обтекании тела, мало отличающегося от цилиндрического.

В случае стационарного течения можно задать

$$
\psi=\sum_{n=1}^{\infty} r^{\lambda_{n}}\left(A_{n} \cos \lambda_{n} \theta+B_{n} \sin \lambda_{n} \theta\right)
$$

где $A_{n}, B_{n}$ - произвольные константы. При $\lambda_{n}<0$ решение можно применять для описания течений, возникающих при внешнем обтекании, при $\lambda_{n}>0$ решение можно использовать для описания течений в каналах.

Условия на фронте ударной волны $x^{0}=x^{0}\left(r, \theta, t^{0}\right)$ получим из условий Ренкина-Гюгонио, подставляя в них разложение (2.11) и оставляя члены старшего порядка:

$$
\begin{aligned}
2 \frac{\partial x^{0}}{\partial t^{0}}+\left(\frac{\partial x^{0}}{\partial r}\right)^{2}+\frac{1}{r^{2}}\left(\frac{\partial x^{0}}{\partial \theta}\right)^{2}+2 \psi_{r} \frac{\partial x^{0}}{\partial r}+\frac{2}{r^{2}} \psi_{\theta} \frac{\partial x^{0}}{\partial \theta}= & \\
& =\frac{\chi-1}{2}\left(2 \psi_{t^{0}}+\psi_{r}^{2}+\frac{1}{r^{2}} \psi_{\theta}^{2}\right)+\frac{\chi+1}{2}\left(\varphi_{x^{0}}+\varphi_{x^{0}}^{*}\right), \quad \varphi=\varphi^{*} .
\end{aligned}
$$

Здесь $\varphi$ и $\varphi^{*}$ соответствуют течению с разных сторон от ударной волны. Если в (2.14) положить $\varphi \equiv \varphi^{*}$, то получим характеристическое уравнение для $(2.12)$.

Выведем условия на обтекаемой поверхности, мало отличающейся от цилиндрической, задав ее в виде

$$
r=r_{0}\left(\theta, t^{0}\right)+r_{2}\left(x^{0}, \theta, t^{0}\right) \varepsilon^{4}+\ldots
$$

Подставляя (2.11) и (2.15) в точное условие непротекания

$$
-\Phi_{x} r_{x}+\Phi_{r}-r^{-2} r_{\theta} \Phi_{\theta}=r_{t}
$$

и оставляя старшие члены, получим

$$
\psi_{r}-\frac{1}{r_{0}^{2}} \frac{\partial r_{0}}{\partial \theta} \psi_{\theta}=\frac{\partial r_{0}}{\partial t}, \quad \varphi_{r}-\frac{1}{r_{0}^{2}} \frac{\partial r_{0}}{\partial \theta} \varphi_{\theta}=\frac{\partial r_{2}}{\partial x^{0}} .
$$

Здесь значения $\varphi_{r}, \varphi_{\theta}, \psi_{r}, \psi_{\theta}$ вычисляются при $r=r_{0}\left(\theta, t^{0}\right)$.

Уравнение звуковой поверхности $\left(V^{2}=a^{2}\right)$ в трансзвуковом приближении принимает вид

$$
N \equiv \frac{\chi+1}{2}\left(\psi_{r}^{2}+\frac{1}{r^{2}} \psi_{\theta}^{2}\right)+(\chi-1) \psi_{t^{0}}+(\chi+1) \varphi_{x^{0}}=0 .
$$

Для установившихся течений уравнение (2.12) имеет смешанный тип. В этом случае звуковая поверхность $N=0$ является поверхностью параболичности уравнения (2.12), при этом в сверхзвуковой области (области гиперболичности) $N>0$, в дозвуковой области (области эллиптичности) $N<0$.

Подставляя (2.11) в выражение для давления (2.10), проводя разложение в ряд Тейлора и оставляя старшие по порядку члены, получим асимптотическую формулу для определения давления:

$$
P=1-\chi \varepsilon^{2}\left(\psi_{t^{0}}+\varphi_{x^{0}}+\frac{1}{2} \psi_{r}^{2}+\frac{1}{2 r^{2}} \psi_{\theta}^{2}\right) .
$$




\section{3. НЕКОТОРЫЕ ЧАСТНЫЕ РЕШЕНИЯ ТРАНСЗВУКОВЫХ УРАВНЕНИЙ}

3.1. Решения классического трансзвукового уравнения. Уравнение (2.13) в декартовой системе координат имеет вид (индекс 0 у переменных $x, t$ будем здесь и далее опускать):

$$
2 \varphi_{x t}+(\chi+1) \varphi_{x} \varphi_{x x}-\varphi_{y y}-\varphi_{z z}=0 .
$$

Уравнение (3.1) равносильно системе уравнений

$$
2 u_{t}+(\chi+1) u u_{x}-v_{y}-w_{z}, \quad u_{y}=v_{x}, \quad u_{z}=w_{x}, \quad v_{z}=w_{y}
$$

где $u, v, w$-проекции вектора скорости на оси декартовой системы координат $x, y, z\left(u=\varphi_{x}\right.$, $\left.v=\varphi_{y}, w=\varphi_{z}\right)$.

Рассмотрим систему (3.2) для плоских течений $\left(w=\varphi_{z}=0\right)$. Считая $u, v, x, y$ функциями параметров $\xi, \eta, t$, систему (3.2) запишем в параметрическом виде:

$$
\left\{\begin{array}{c}
2 u_{t}\left(x_{\xi} y_{\eta}-x_{\eta} y_{\xi}\right)-2 u_{\xi}\left(x_{t} y_{\eta}-x_{\eta} y_{t}\right)-2 u_{\eta}\left(x_{\xi} y_{t}-x_{t} y_{\xi}\right)+ \\
\quad+(\chi+1) u\left(u_{\xi} y_{\eta}-u_{\eta} y_{\xi}\right)-v_{\eta} x_{\xi}+v_{\xi} x_{\eta}=0 \\
u_{\eta} x_{\xi}-u_{\xi} x_{\eta}-v_{\xi} y_{\eta}+v_{\eta} y_{\xi}=0 .
\end{array}\right.
$$

Решение системы (3.3) будем искать в виде:

$$
u=\sum_{k=0}^{\alpha} U_{k} \eta^{k}, \quad v=\sum_{k=0}^{\beta} V_{k} \eta^{k}, \quad x=\sum_{k=0}^{\gamma} X_{k} \eta^{k}, \quad y=\sum_{k=0}^{\omega} Y_{k} \eta^{k} .
$$

Здесь $U_{k}, V_{k}, X_{k}, Y_{k}$ - функции, зависящие от $\xi, t ; \alpha, \beta, \gamma, \omega$-натуральные числа. Число уравнений $s$, полученных при подстановке (3.4) в (3.3), равно

$$
s=\max (2 \alpha+\omega-1, \beta+\gamma-1, \alpha+\gamma+\omega-1)+\max (\alpha+\gamma-1, \beta+\omega-1)+2 .
$$

Для того, чтобы система была определенной, необходимо выполнение условия

$$
j=r-s \geqslant 0,
$$

где $r=\alpha+\beta+\gamma+\omega+4$ - число функций. Получены все возможные значения $\alpha, \beta, \gamma, \omega \in N$, для которых система 3.3 будет определенной или недоопределенной. В частности, при $j=3$ возможны следующие четверки значений $(\alpha, \beta, \gamma, \omega)$ :

$$
\begin{array}{lllll}
(0,1,2,1), & (1,2,2,1), & (2,3,2,1), & (0,0,1,1), & (0,1,1,1), \\
(1,1,1,1), & (1,2,1,1), & (0,0,0,1), & (0,1,0,1), & (0,0,1,0),
\end{array}
$$

$$
(0,1,1,0), \quad(1,1,1,0), \quad(1,2,1,0), \quad(2,3,1,0) .
$$

Решения с $\omega=1$ являются решениями по степеням $y$. Среди таких решений отметим решения

$$
u=U_{0}+U_{2} y^{2}, \quad v=V_{1} y+V_{3} y^{3}, \quad x=X_{0}+X_{2} y^{2} .
$$

Функции $U_{k}, V_{k}, X_{k}$ зависят в общем случае от $\xi, t$. В случае, когда $U_{2}, V_{3}, X_{2}$ зависят только от $t$, решение (3.5) описывает течение в соплах Лаваля с местными сверхзвуковыми зонами (см. [27]).

Уравнение 3.1 имеет решения вида

$$
\varphi=\sum_{k=0}^{3} \varphi_{k}(y, z, t) x^{k} .
$$

Отметим еще несколько классов решений для уравнений (2.13), (3.1):

$$
\begin{gathered}
\varphi=t^{2 n-1} \varphi^{*}\left(x^{*}, y^{*}, z^{*}\right), \quad x^{*}=x t^{-n}, \quad y^{*}=y t^{-\frac{n+1}{2}}, \quad z^{*}=z t^{-\frac{n+1}{2}}, \\
\varphi=e^{4 n t} \varphi^{*}\left(x^{*}, y^{*}, z^{*}\right), \quad x^{*}=x e^{-2 n t}, \quad y^{*}=y e^{-n t}, \quad z^{*}=z e^{-n t} . \\
\varphi=e^{-3 a y} g(\xi, z), \quad \xi=x^{*} e^{a y} \\
\varphi=r^{-2} g(\xi, \theta), \quad \xi=r e^{a x^{*}+b \theta} \\
\varphi=g(\xi, r)+\beta\left(x^{*}, r, t\right), \quad \xi=x^{*}+a \theta+b t .
\end{gathered}
$$




\section{2. Частные решения трансзвукового уравнения с поперечным возмущением и их приложения.}

3.2.1. Решения полиномиального вида. Уравнение (2.12) имеет решение полиномиального вида (индекс 0 у переменных $x, t$ будем здесь и далее опускать):

$$
\varphi=\sum_{k=0}^{3} \varphi_{k}(r, \theta, t) x^{k} .
$$

Четыре функции $\varphi_{0}, \varphi_{1}, \varphi_{2}, \varphi_{3}$ удовлетворяют следующей системе из четырех уравнений:

$$
\left\{\begin{array}{l}
18 \varphi_{3}^{2}(\chi+1)=\Delta \varphi_{3}, \\
6 \varphi_{3 t}+18(\chi+1) \varphi_{2} \varphi_{3}+6 \psi_{r} \varphi_{3 r}+\frac{6}{r^{2}} \psi_{\theta} \varphi_{3 \theta}=\Delta \varphi_{2}, \\
4 \varphi_{2 t}+(\chi+1)\left(6 \varphi_{1} \varphi_{3}+4 \varphi_{2}^{2}\right)+4 \psi_{r} \varphi_{2 r}+\frac{4}{r^{2}} \psi_{\theta} \varphi_{2 \theta}+6(\chi-1) \varphi_{3}\left(\psi_{t}+\frac{1}{2} \psi_{r}^{2}+\frac{1}{2 r^{2}} \psi_{\theta}^{2}\right)=\Delta \varphi_{1}, \\
2 \varphi_{1 t}+2(\chi+1) \varphi_{1} \varphi_{2}+2 \psi_{r} \varphi_{1 r}+\frac{2}{r^{2}} \psi_{\theta} \varphi_{1 \theta}+2(\chi-1) \varphi_{2}\left(\psi_{t}+\frac{1}{2} \psi_{r}^{2}+\frac{1}{2 r^{2}} \psi_{\theta}^{2}\right)-L(\psi)=\Delta \varphi_{0} .
\end{array}\right.
$$

В частных случаях функции $\varphi_{0}, \varphi_{1}, \varphi_{2}, \varphi_{3}, \psi$ могут зависеть от $(r, t)$, от $(r, \theta)$ или только от $r$. Уравнение для $\varphi_{3}$ - нелинейное. Если $\varphi_{3}=0$, то система уравнений для $\varphi_{0}, \varphi_{1}, \varphi_{2}$ становится линейной.

3.2.2. Автомодельные решения. Уравнение (2.12) для нестационарных течений имеет автомодельные решения

$$
\psi=t^{\beta} \bar{\psi}(\zeta, \eta), \quad \varphi=t^{2 \beta-1} \bar{\varphi}(\xi, \zeta, \eta), \quad \xi=\frac{x}{t^{\beta}}, \quad \zeta=\frac{r}{t^{(\beta+1) / 2}}, \quad \eta=\theta+\alpha \ln t,
$$

где $\alpha, \beta$ - произвольные числа. В стационарном случае уравнение (2.12) допускает класс решений

$$
\psi=r^{\beta} \bar{\psi}(\theta), \quad \varphi=r^{3 \beta-2} \bar{\varphi}(\xi, \theta), \quad \xi=\frac{x}{r^{\beta}},
$$

где $\beta$-произвольное число. Переход к автомодельным переменным позволяет уменьшить число независимых переменных от четырех до трех в нестационарном случае и от трех до двух в стационарном случае.

3.2.3. Течения в неограниченной области. Такие решения должны удовлетворять условиям затухания возмущений вдали от тела (на бесконечности), например, при $r \rightarrow \infty$. Укажем решение для случаев $\psi=\Gamma \theta, \psi=Q \ln r$, обладающее следующими свойствами: $V_{x}, V_{r}, V \rightarrow 0$ при $r \rightarrow \infty$ (где $V_{(x)}=\varphi_{x}, V_{(r)}=\varphi_{r}, V_{(\theta)}=\varphi_{\theta} / r$ - проекции вектора скорости):

$$
\varphi=f(x, \theta) r^{-2}+g(r, \theta), \quad \Delta g=0 .
$$

3.2.4. Осесимметричные стачионарные течения в соплах Лаваля. В классе решений (3.6) в случае установившихся течений содержится решение, которое описывает течение газа в соплах Лаваля с постоянным ускорением $\left(\varphi_{x x}=\right.$ const $)$ и учитывает закрутку потока $(\psi=\Gamma \theta$, $\Gamma=$ const $)$ :

$$
\varphi=a x^{2}+(\chi+1) a^{2} r^{2} x+\left[\frac{\chi-1}{2} \Gamma^{2} a \ln ^{2} r+\frac{1}{8}(\chi+1)^{2} a^{3} r^{4}\right] .
$$

Уравнение звуковой поверхности для (3.7), согласно (2.17), имеет вид

$$
x=-\frac{\chi+1}{2} a r^{2}-\frac{\Gamma^{2}}{4 a r^{2}} .
$$

Условия (2.16) принимают вид:

$$
\frac{\partial r_{0}}{\partial \theta}=0, \quad \frac{\partial r_{2}}{\partial x}=\varphi_{r}
$$




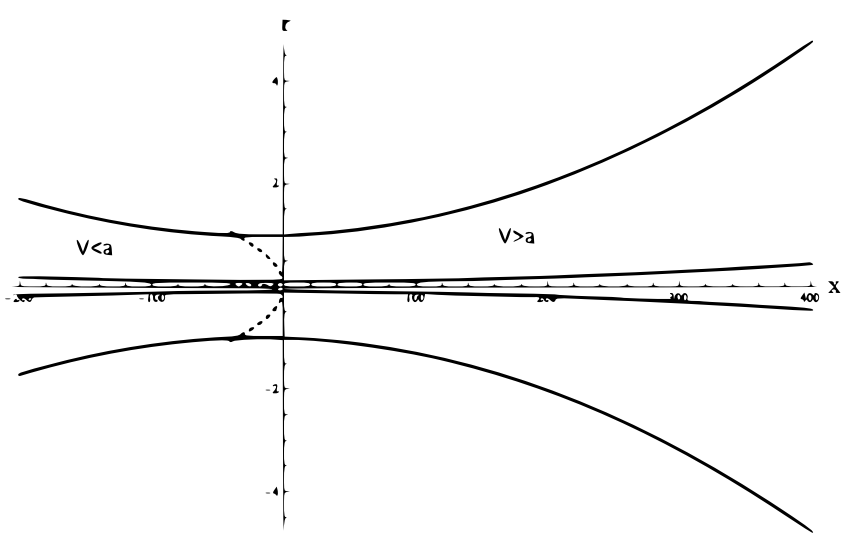

Рис. 1. Продольное сечение сопла.

( $\varphi_{r}$ вычисляется при $\left.r=r_{0}(\theta)\right)$. Тогда получим уравнение обтекаемой поверхности:

$$
r=r_{0}+\epsilon^{4}\left[r_{0} a^{2}(\chi+1) x^{2}+\left((\chi-1) \Gamma^{2} a \frac{\ln r_{0}}{r_{0}}+\frac{1}{2}(\chi+1)^{2} a^{3} r_{0}^{3}\right) x\right], \quad r_{0}=\text { const } .
$$

Решение (3.7) описывает течения типа течений Майера в кольцеобразных соплах, уравнения внутренней и внешней стенок которых получим из (3.8) при $r_{0}=r_{0}^{(1)}, r_{0}=r_{0}^{(2)}, r_{0}^{(k)}=$ const $\neq 0$. В качестве примера на рис. 1 изображены стенки сопла и звуковая поверхность, соответствующие решению (3.7), при $r_{0}^{(1)}=0,1, r_{0}^{(2)}=1, \Gamma=1, \chi=1,4, a=30, \varepsilon=0,1$. Левее звуковой поверхности (на которой $V=a$ ) скорость потока дозвуковая $(V<a)$, правее - сверхзвуковая $(V>a)$; при этом реализуется течение Майера с переходом через скорость звука по всей горловине сопла.

Если в (3.7) положить $\Gamma=0$, то получим известное решение, описывающее течение Майера в центре сопла Лаваля.

3.2.5. Нестационарные течения в каналах между вращающимися плоскостями. Рассмотрим случай, соответствующий движению газа между вращающимися плоскостями $\theta=\theta_{1}(t), \theta=\theta_{2}(t)$. В этом случае

$$
\psi=r^{2}(a(t) \cos 2 \theta+b(t) \sin 2 \theta)=r^{2} g(\theta, t) ;
$$

при этом в $(2.12) L(\psi)=r^{2} G(\theta, t)$,

$$
\begin{aligned}
G(\theta, t)=8\left(a^{\prime} a+b^{\prime} b\right)+8(a \cos 2 \theta+b \sin 2 \theta)\left(2 a b \sin 4 \theta+a^{2} \cos 4 \theta\right. & \left.-b^{2} \cos 4 \theta\right)+ \\
& +a^{\prime \prime} \cos 2 \theta+b^{\prime \prime} \sin 2 \theta .
\end{aligned}
$$

Подставляя (3.9) в (2.12), получим уравнение для $\varphi(x, r, \theta, t)$ :

$$
\begin{aligned}
2 \varphi_{x t}+(\chi+1) \varphi_{x} \varphi_{x x}+4 r(a & \cos 2 \theta+b \sin 2 \theta) \varphi_{x r}+4(b \cos 2 \theta-a \sin 2 \theta) \varphi_{x \theta}-\Delta \varphi+ \\
+(\chi-1) r^{2}\left(a^{\prime} \cos 2 \theta+b^{\prime} \sin 2 \theta+2 a^{2}+2 b^{2}\right) \varphi_{x x} & =-r^{2} G(\theta, t) .
\end{aligned}
$$

Уравнение (3.10) допускает решение вида (3.6). Тогда получим следующую систему четырех уравнений для функций $\varphi_{0}(r, \theta, t), \varphi_{1}(r, \theta, t), \varphi_{2}(r, \theta, t)$ и $\varphi_{3}(r, \theta, t)$ :

$$
\left\{\begin{array}{l}
18(\chi+1) \varphi_{3}^{2}=\Delta \varphi_{3}, \\
6 \varphi_{3 t}+18(\chi+1) \varphi_{2} \varphi_{3}+12(b \cos 2 \theta-a \sin 2 \theta) \varphi_{3 \theta}+12 r(a \cos 2 \theta+b \sin 2 \theta) \varphi_{3 r}=\Delta \varphi_{2}, \\
4 \varphi_{2 t}+(\chi+1)\left(6 \varphi_{1} \varphi_{3}+4 \varphi_{2}^{2}\right)+8 r(a \cos 2 \theta+b \sin 2 \theta) \varphi_{2 r}+8(b \cos 2 \theta-a \sin 2 \theta) \varphi_{2 \theta}+ \\
\quad+6(\chi-1) r^{2} \varphi_{3}\left(a^{\prime} \cos 2 \theta+b^{\prime} \sin 2 \theta+2 a^{2}+2 b^{2}\right)=\Delta \varphi_{1}, \\
2 \varphi_{1 t}+2(\chi+1) \varphi_{1} \varphi_{2}+4 r(a \cos 2 \theta+b \sin 2 \theta) \varphi_{1 r}+4(b \cos 2 \theta-a \sin 2 \theta) \varphi_{1 \theta}+ \\
\quad+2(\chi-1) r^{2}\left(a^{\prime} \cos 2 \theta+b^{\prime} \sin 2 \theta+2 a^{2}+2 b^{2}\right) \varphi_{2}-r^{2} G(\theta, t)=\Delta \varphi_{0} .
\end{array}\right.
$$




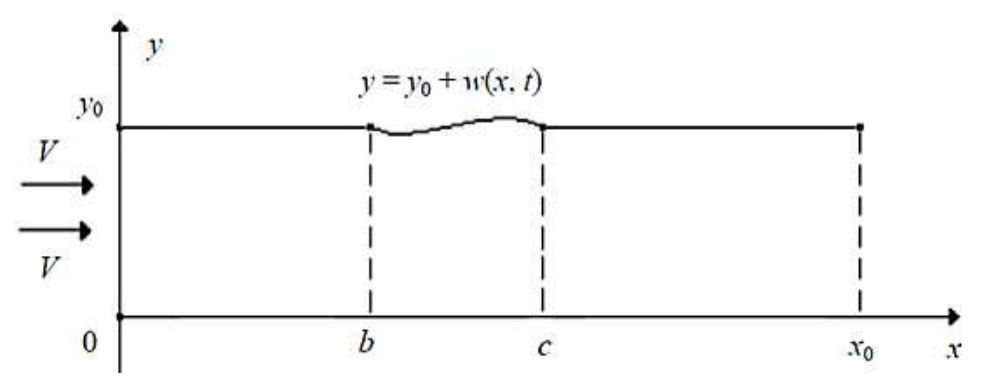

Рис. 2. Канал, стенка которого содержит деформируемый элемент.

Система (3.11) допускает следующее частное решение:

$$
\varphi_{3}(r, \theta, t)=r^{-2} f_{3}(\theta, t), \quad \varphi_{2}(r, \theta, t)=f_{2}(\theta, t), \quad \varphi_{1}(r, \theta, t)=r^{2} f_{1}(\theta, t), \quad \varphi_{0}(r, \theta, t)=r^{4} f_{0}(\theta, t) .
$$

В случае $f_{3}(\theta, t)=0$ решение (3.6) принимает вид

$$
\varphi(x, r, \theta, t)=f_{2}(\theta, t) x^{2}+f_{1}(\theta, t) r^{2} x+f_{0}(\theta, t) r^{4} .
$$

Это решение, удовлетворяющее условию конечности скорости на оси $r=0$, описывает течения в каналах. Согласно 3.12, получим систему для трех функций $f_{0}(\theta, t), f_{1}(\theta, t), f_{2}(\theta, t)$ :

$$
\left\{\begin{array}{l}
f_{2 \theta \theta}=0, \\
4 f_{1}+f_{1 \theta \theta}=4 f_{2 t}+4(\chi+1) f_{2}^{2}+8(b \cos 2 \theta-a \sin 2 \theta) f_{2 \theta}, \\
16 f_{0}+f_{0 \theta \theta}=2 f_{1 t}+2(\chi+1) f_{1} f_{2}+4(b \cos 2 \theta-a \sin 2 \theta) f_{1 \theta}+8(a \cos 2 \theta+b \sin 2 \theta) f_{1}+ \\
\quad+2(\chi-1)\left(a^{\prime} \cos 2 \theta+b^{\prime} \sin 2 \theta+2 a^{2}+2 b^{2}\right) f_{2}-G(\theta, t) .
\end{array}\right.
$$

Общее решение системы (3.13) несложно записать. Граничные условия для $g(\theta, t), f_{n}(\theta, t), n=$ $0,1,2$ на плоскостях $\theta=\theta_{k}(t), k=1,2$, получим, подставляя (3.9) и (3.12) в (2.11) и затем удовлетворяя условию непротекания $\Phi_{\theta}\left(x, r, \theta_{k}(t), t\right)=r^{2} \theta_{k}^{\prime}(t)$ :

$$
\begin{aligned}
\frac{\partial g}{\partial \theta}\left(\theta_{k}(t), t\right) & =\theta_{k}^{\prime}(t), \quad k=1,2 \\
\frac{\partial f_{n}}{\partial \theta}\left(\theta_{k}(t), t\right) & =0, \quad k=1,2
\end{aligned}
$$

Функции $a(t), b(t)$ в (3.9) определяются согласно (3.14):

$$
a(t)=\frac{1}{A}\left(\theta_{2}^{\prime} \cos 2 \theta_{2}-\theta_{1}^{\prime} \cos 2 \theta_{1}\right), \quad b(t)=\frac{1}{A}\left(\theta_{2}^{\prime} \sin 2 \theta_{1}-\theta_{1}^{\prime} \sin 2 \theta_{2}\right), \quad A=2 \sin 2\left(\theta_{1}-\theta_{2}\right) .
$$

Из условий (3.15) определяются произвольные функции, зависящие от $t$ и входящие в выражения для $f_{n}(\theta, t), n=0,1,2$.

\section{4. ИССЛЕДОВАНИЕ ДИНАМИЧЕСКОЙ УСТОЙЧИВОСТИ УПРУГОГО ЭЛЕМЕНТА СТЕНКИ КАНАЛА}

Эта задача рассматривается в качестве примера применения линейного уравнения (2.4).

4.1. Постановка задачи. Рассмотрим плоское течение в прямолинейном канале

$$
J=\left\{(x, y) \in \mathbb{R}^{2}: 0<x<x_{0}, 0<y<y_{0}\right\} .
$$

Скорость невозмущенного однородного потока равна $V$ и направлена вдоль оси $O x$. Упругой является часть стенки $y=y_{0}$ при $x \in[b, c]$ (рис. 2). Канал с упругим элементом моделирует вибрационное устройство, предназначенное для интенсификации технологических процессов, например, для размешивания (за счет автоколебаний) и приготовления однородных смесей. Введем обозначения: $w(x, t)$ - функция деформации упругого элемента стенки канала; $\varphi(x, y, t)-$ потенциал скорости возмущенного потока. 
Математическая постановка задачи имеет следующий вид:

$$
\begin{gathered}
\varphi_{t t}+2 V \varphi_{x t}+V^{2} \varphi_{x x}=a^{2}\left(\varphi_{x x}+\varphi_{y y}\right), \quad(x, y) \in J, \quad t \geqslant 0, \\
\varphi_{y}\left(x, y_{0}, t\right)=\dot{w}(x, t)+V w^{\prime}(x, t), \quad x \in(b, c), \quad t \geqslant 0, \\
\varphi_{y}\left(x, y_{0}, t\right)=0, \quad x \in(0, b] \cup\left[c, x_{0}\right), \quad t \geqslant 0, \\
\varphi_{y}(x, 0, t)=0, \quad x \in\left(0, x_{0}\right], \quad t \geqslant 0, \\
\varphi(0, y, t)=0, \quad \varphi\left(x_{0}, y, t\right)=0, \quad y \in\left(0, y_{0}\right), \quad t \geqslant 0, \\
D w^{\prime \prime \prime \prime}(x, t)+\beta_{2} \dot{w}^{\prime \prime \prime \prime}(x, t)+M \ddot{w}(x, t)+N w^{\prime \prime}(x, t)+\beta_{1} \dot{w}(x, t)+\beta_{0} w(x, t)= \\
=-\rho\left(\varphi_{t}\left(x, y_{0}, t\right)+V \varphi_{x}\left(x, y_{0}, t\right)\right), \quad x \in(b, c), \quad t \geqslant 0, \\
w(b, t)=w^{\prime \prime}(b, t)=w(c, t)=w^{\prime \prime}(c, t)=0, \quad t \geqslant 0, \\
w(x, 0)=f_{1}(x), x \in(b, c), \quad \dot{w}(x, 0)=f_{2}(x), x \in(b, c), \\
\varphi(x, y, 0)=\psi_{1}(x, y),(x, y) \in J, \quad \varphi_{t}(x, y, 0)=\psi_{2}(x, y), \quad(x, y) \in J .
\end{gathered}
$$

Индексы $x, y, t$ снизу обозначают частные производные по $x, y, t$; штрих и точка сверху - частные производные по $x$ и $t$ соответственно; $\rho$ - плотность жидкости в однородном невозмущенном потоке; $D, M$ - изгибная жесткость и погонная масса пластины; $N$ - сжимающая $(N>0)$ или растягивающая $(N<0)$ пластину сила; $\beta_{1}, \beta_{2}$-коэффициенты внешнего и внутреннего демпфирования; $\beta_{0}$ - коэффициент жесткости основания; $a$-скорость звука в невозмущенном потоке жидкости $(a>V)$.

Уравнение (4.1) описывает динамику идеального сжимаемого газа (жидкости), уравнение (4.6) описывает динамику упругого элемента стенки канала, (4.2), (4.3), (4.4) - условия непротекания, (4.5) - условия отсутствия возмущений в граничных сечениях канала, (4.7) - условия шарнирного закрепления концов упругого элемента, (4.8), (4.9) - начальные условия.

4.2. Исследование устойчивости. Исследуем устойчивость нулевого решения $\varphi(x, y, t) \equiv 0$, $w(x, t) \equiv 0$ краевой задачи (4.1)-(4.7) по отношению к возмущениям начальных условий (4.8)(4.9), т.е. устойчивость по Ляпунову. Введем функционал

$$
\begin{aligned}
\Phi(t)=\iint_{J}\left(\varphi_{t}^{2}+\right. & \left.\left(a^{2}-V^{2}\right) \varphi_{x}^{2}+a^{2} \varphi_{y}^{2}\right) d x d y- \\
& -2 a^{2} V \int_{b}^{c} \phi\left(x, y_{0}, t\right) w^{\prime}(x, t) d x+\frac{a^{2}}{\rho} \int_{b}^{c}\left(M \dot{w}^{2}+D w^{\prime \prime 2}-N w^{\prime 2}+\beta_{0} w^{2}\right) d x .
\end{aligned}
$$

Для функций $\varphi(x, y, t)$ и $w(x, t)$, удовлетворяющих уравнениям $(4.1)$ и $(4.6)$, производная от $\Phi$ по $t$ примет вид

$$
\begin{array}{r}
\dot{\Phi}(t)=2 \iint_{J}\left(\varphi_{t}\left(-2 V \varphi_{x t}-V^{2} \varphi_{x x}+a^{2}\left(\varphi_{x x}+\varphi_{y y}\right)\right)+\left(a^{2}-V^{2}\right) \varphi_{x} \varphi_{x t}+a^{2} \varphi_{y} \varphi_{y t}\right) d x d y- \\
-2 a^{2} V \int_{b}^{c}\left(\phi_{t}\left(x, y_{0}, t\right) w^{\prime}(x, t)+\phi\left(x, y_{0}, t\right) \dot{w}^{\prime}(x, t)\right) d x+ \\
+\frac{2 a^{2}}{\rho} \int_{b}^{c}\left(\dot{w}\left[-\rho\left(\varphi_{t}\left(x, y_{0}, t\right)+V \varphi_{x}\left(x, y_{0}, y\right)\right)-D w^{\prime \prime \prime \prime}-\beta_{2} \dot{w}^{\prime \prime \prime \prime}-N w^{\prime \prime}-\beta_{1} \dot{w}-\beta_{0} w\right]+\right. \\
\left.+D w^{\prime \prime} \dot{w}^{\prime \prime}-N w^{\prime} \dot{w}^{\prime}+\beta_{0} w \dot{w}\right) d x .
\end{array}
$$


Произведя интегрирование с учетом условий (4.2)-(4.5) и (4.7), получим

$$
\dot{\Phi}(t)=-\frac{2 a^{2}}{\rho} \int_{b}^{c}\left(\beta_{2} \dot{w}^{\prime \prime 2}+\beta_{1} \dot{w}^{2}\right) d x .
$$

Пусть выполняются условия

$$
\beta_{2} \geqslant 0, \quad \beta_{1} \geqslant 0
$$

тогда имеют место неравенства

$$
\dot{\Phi}(t) \leqslant 0 \quad \Rightarrow \quad \Phi(t) \leqslant \Phi(0) .
$$

Для оценки функционала для функции $w(x, t)$ запишем неравенства Рэлея (см. [35]):

$$
\begin{gathered}
\int_{b}^{c} w^{\prime \prime 2}(x, t) d x \geqslant \lambda_{1} \int_{b}^{c} w^{\prime 2}(x, t) d x, \quad \int_{b}^{c} w^{\prime \prime 2}(x, t) d x \geqslant \mu_{1} \int_{b}^{c} w^{2}(x, t) d x . \\
\int_{0}^{x_{0}} \varphi_{x}^{2} d x \geqslant \eta_{1} \int_{0}^{x_{0}} \varphi^{2} d x,
\end{gathered}
$$

где $\lambda_{1}, \mu_{1}$ - наименьшие собственные значения краевых задач

$$
\psi^{\prime \prime \prime \prime}(x)=-\lambda \psi^{\prime \prime}(x), \quad \psi^{\prime \prime \prime \prime}(x)=\mu \psi(x), \quad x \in(b, c),
$$

с граничными условиями $(4.7) ; \eta_{1}=\pi^{2} / x_{0}^{2}$ - наименьшее собственное значение краевой задачи $\psi^{\prime \prime}=-\eta \psi, x \in\left(0, x_{0}\right)$, с краевыми условиями $\psi(0)=0, \psi\left(x_{0}\right)=0$, которые соответствуют (4.5). Интегрируя неравенство (4.16) от 0 до $y_{0}$ по переменной $y$, получим

$$
\iint_{J} \varphi_{x}^{2}(x, y, t) d x d y \geqslant \frac{\pi^{2}}{x_{0}^{2}} \iint_{J} \varphi^{2}(x, y, t) d x d y .
$$

Воспользовавшись неравенством Коши-Буняковского, получим неравенства

$$
\begin{gathered}
\left(\int_{b}^{x} w^{\prime}(x, t) d x\right)^{2} \leqslant \int_{b}^{x} 1^{2} d x \int_{b}^{x} w^{\prime 2}(x, t) d x \leqslant \int_{b}^{c} 1^{2} d x \int_{b}^{c} w^{\prime 2}(x, t) d x \\
\left(\left.w(x, t)\right|_{b} ^{x}\right)^{2} \leqslant\left(\left.x\right|_{b} ^{c}\right) \int_{b}^{c} w^{\prime 2} d x, \quad w^{2}(x, t) \leqslant(c-b) \int_{b}^{c} w^{\prime 2}(x, t) d x \\
\iint_{J} \varphi_{y}^{2} d x d y \geqslant \frac{2}{y_{0}^{2}} \iint_{J}\left(\varphi\left(x, y_{0}, t\right)-\varphi(x, y, t)\right)^{2} d x d y .
\end{gathered}
$$

Оценим $\Phi(0)$ сверху, используя неравенства (4.15) и очевидное неравенство $-2 a b \leqslant a^{2}+b^{2}$ :

$$
\begin{aligned}
\Phi(0) \leqslant \iint_{J}\left(\varphi_{t 0}^{2}+\left(a^{2}-V^{2}\right) \varphi_{x 0}^{2}+a^{2} \varphi_{y 0}^{2}\right) d x & d y+a^{2} \int_{b}^{c} \varphi^{2}\left(x, y_{0}, 0\right) d x+ \\
& +\frac{a^{2}}{\rho} \int_{b}^{c}\left(M \dot{w}_{0}^{2}+\left(D+\frac{|N|+\rho V^{2}}{\lambda_{1}}+\frac{\beta_{0}}{\mu_{1}}\right) w_{0}^{\prime \prime 2}\right) d x
\end{aligned}
$$

Здесь введены обозначения $\varphi_{t 0}=\varphi_{t}(x, y, 0), \varphi_{x, 0}=\varphi_{x}(x, y, 0), \varphi_{y 0}=\varphi_{y}(x, y, 0), \dot{w}_{0}=\dot{w}(x, 0)$, $w_{0}=w(x, 0), w_{0}^{\prime}=w^{\prime}(x, 0), w_{0}^{\prime \prime}=w^{\prime \prime}(x, 0)$. 
Оценим $\Phi(t)$ снизу, применяя (4.15), (4.17), (4.20) к (4.10):

$$
\begin{aligned}
\Phi(t) \geqslant \iint_{J}\left(\varphi_{t}^{2}+\left(a^{2}-V^{2}\right) \frac{\pi^{2}}{x_{0}^{2}} \varphi^{2}\right. & \left.+\frac{a^{2}}{y_{0}^{2}}\left(\varphi\left(x, y_{0}, t\right)-\varphi(x, y, t)\right)^{2}\right) d x d y- \\
& -2 a^{2} V \int_{b}^{c} \varphi\left(x, y_{0}, t\right) w^{\prime}(x, t) d x+\frac{a^{2}}{\rho} \int_{b}^{c}\left(\lambda_{1} D-N\right) w^{\prime 2} d x .
\end{aligned}
$$

Введем обозначение

$$
f(x, t)= \begin{cases}0, & x \in(0, b], \\ w^{\prime}(x, t), & x \in(b, c), \\ 0, & x \in\left[c, x_{0}\right),\end{cases}
$$

Тогда из (4.22) получим неравенство

$$
\begin{array}{r}
\Phi(t) \geqslant \iint_{J}\left[\varphi_{t}^{2}(x, y, t)+\left(\left(a^{2}-V^{2}\right) \frac{\pi^{2}}{x_{0}^{2}} \varphi^{2}(x, y, t)+\frac{a^{2}}{y_{0}^{2}}\right) \varphi^{2}(x, y, t)-\frac{4 a^{2}}{y_{0}^{2}} \varphi\left(x, y_{0}, t\right) \varphi(x, y, t)+\right. \\
\left.+\frac{2 a^{2}}{y_{0}^{2}} \varphi^{2}\left(x, y_{0}, t\right)-\frac{2 a^{2} V}{y_{0}} \varphi\left(x, y_{0}, t\right) f(x, t)+\frac{a^{2}\left(\lambda_{1} D-N\right)}{\rho y_{0}} f^{2}(x, t)\right] d x d y .
\end{array}
$$

Согласно критерию Сильвестра квадратичная форма относительно $\varphi(x, y, t), \varphi\left(x, y_{0}, t\right), f(x, t)$ в (4.23) будет положительно определенной, если выполняются условия

$$
\begin{gathered}
\lambda_{1} D-N>0 \\
\frac{\lambda_{1} D-N}{\rho y_{0}} \cdot \frac{2\left(a^{2}-V^{2}\right) \pi^{2}}{x_{0}^{2}}-V^{2}\left(\frac{\left(a^{2}-V^{2}\right) \pi^{2}}{x_{0}^{2}}+\frac{2 a^{2}}{y_{0}^{2}}\right)>0 .
\end{gathered}
$$

Преобразуем неравенство (4.25):

$$
N<\lambda_{1} D-\frac{V^{2} x_{0}^{2} \rho y_{0}}{2\left(a^{2}-V^{2}\right) \pi^{2}}\left(\frac{\left(a^{2}-V^{2}\right) \pi^{2}}{x_{0}^{2}}+\frac{2 a^{2}}{y_{0}^{2}}\right) .
$$

Оценивая квадратичную форму в (4.23) относительно $w(x, t)$ с учетом $(4.18)$, получим

$$
\Phi(t) \geqslant \frac{\Delta_{3} y_{0}}{\Delta_{2}(c-b)} w^{2}(x, t)
$$

где $\Delta_{2}=d_{11} d_{22}-d_{12}^{2}>0, \Delta_{3}=d_{33} \Delta_{2}-d_{23}^{2} d_{11}>0$,

$$
d_{11}=\frac{\left(a^{2}-V^{2}\right) \pi^{2}}{x_{0}^{2}}+\frac{2 a^{2}}{y_{0}^{2}}, \quad d_{22}=d_{12}=\frac{2 a^{2}}{y_{0}^{2}}, \quad d_{23}=\frac{V}{y_{0}^{2}}, \quad d_{33}=\frac{a^{2}\left(\lambda_{1} D-N\right)}{\rho y_{0}} .
$$

Учитывая (4.14), (4.21), (4.27), получим неравенство

$$
\begin{aligned}
w^{2}(x, t) \leqslant \frac{\Delta_{2}(c-b)}{\Delta_{3} y_{0}} \iint_{J}\left(\varphi_{t 0}^{2}+\left(a^{2}-V^{2}\right) \varphi_{x 0}^{2}\right. & \left.+a^{2} \varphi_{y 0}^{2}\right) d x d y+a^{2} \int_{b}^{c} \varphi^{2}\left(x, y_{0}, 0\right) d x+ \\
& +\frac{a^{2}}{\rho} \int_{b}^{c}\left(M \dot{w}_{0}^{2}+\left(D+\frac{|N|+\rho V^{2}}{\lambda_{1}}+\frac{\beta_{0}}{\mu_{1}}\right) w_{0}^{\prime \prime 2}\right) d x
\end{aligned}
$$

из которого вытекает следующая теорема.

Теорема 4.1. Пусть выполняются условия (4.13), (4.24), (4.26). Тогда решение $w(x, t)$ задачи (4.1)-(4.7) устойчиво по отношению $\kappa$ возмущениям начальных данных $\varphi_{t 0}, \varphi_{x 0}, \varphi_{y 0}$, $\varphi\left(x, y_{0}, 0\right), \dot{w}_{0}, w^{\prime \prime}{ }_{0}$. 


\section{СПИСОК ЛИТЕРАТУРЫ}

1. Анкилов А. В., Вельмисов П. А. Устойчивость вязкоупругих элементов стенок проточных каналов. Ульяновск: УлГТУ, 2000.

2. Анкилов А. В., Вельмисов П. А. Динамика и устойчивость упругих пластин при аэрогидродинамическом воздействии. - Ульяновск: УлГТУ, 2009.

3. Анкилов А. В., Вельмисов П. А. Математическое моделирование в задачах динамической устойчивости деформируемых элементов конструкций при аэрогидродинамическом воздействии. - Ульяновск: УлГТУ, 2013.

4. Анкилов А. В., Вельмисов П. А. Устойчивость решений некоторых классов интегро-дифференциальных уравнений в частных производных// Вестн. Самар. гос. ун-та. Сер. естественнонауч. - 2008. 8, № 1 (67). - C. 331-344.

5. Анкилов А. В., Вельмисов П. А. Математическое моделирование динамики и устойчивости упругих элементов крыла// Вестн. Саратов. гос. техн. ун-та. - 2009. - 1, № 37. - С. 7-16.

6. Анкилов A. В., Велвмисов П. А. Об устойчивости решений уравнений взаимодействия упругих стенок каналов с протекающей жидкостью// Вестн. Самар. гос. техн. ун-та. Сер. физ.-мат. науки. - 2011. 1, № 22. - C. 179-185.

7. Анкилов А. В., Вельмисов П. А. Исследование динамики и устойчивости упругого элемента конструкций при сверхзвуковом обтекании// Вестн. Саратов. гос. техн. ун-та. - 2011. - 1, № 3 (57). - С. 59-67.

8. Анкилов А. В., Вельмисов П. А. Функционалы Ляпунова в некоторых задачах динамической устойчивости аэроупругих конструкций. - Ульяновск: УлГТУ, 2015.

9. Анкилов А. В., Вельмисов П. А., Казакова Ю. А. Устойчивость решений одной нелинейной начальнокраевой задачи аэроупругости// Вестн. Самар. гос. техн. ун-та. Сер. физ.-мат. науки. $-2013 .-2$, № 31 . - C. $120-126$.

10. Анкилов А. В., Велвмисов П. А., Семенова Е. П. Исследование динамической устойчивости упругих элементов стенок канала// Вестн. Саратов. гос. техн. ун-та. - 2009. - 1, № 2 (38). - С. 7-17.

11. Анкилов А. В., Велвмисов П. А., Семенова Е. П. О решениях интегро-дифференциальных уравнений в задаче динамики одной аэроупругой системы типа «тандем»// Вестн. Самар. гос. техн. ун-та. Сер. физ.-мат. науки. - 2011. - № 2 (23). - С. 266-271.

12. Анкилов А. В., Вельмисов П. А., Тамарова Ю. А. Математическая модель вибрационного устройства// Автомат. процессов управл. - 2014. - № 3 (37). - С. 58-67.

13. Анкилов А. В., Вельмисов П. А., Тамарова Ю. А. Динамическая устойчивость упругого элемента проточного канала// Изв. вузов. Поволж. регион. Физ.-мат. науки. - 2014. - № 3 (31). - C. 40-55.

14. Барметов Ю. П., Дободейч И. А. К расчету нестационарных течений сжимаемой жидкости в трубопроводе// Изв. вузов. Авиац. техн. - 2006. - № 1. - С. 18-21.

15. Вельмисов П. А. К вопросу о неустановившемся движении газа в соплах Лаваля// Изв. вузов. Мат. - 1976. - № 12. - С. 3-10.

16. Вельмисов П. А. О единственности решения прямой задачи сопла Лаваля// Изв. вузов. Мат. -1979. - № 1. - C. 15-17.

17. Вельмисов П. А. Асимптотическое исследование нелинейных эффектов в задаче о нестационарном сверхзвуковом обтекании профиля// Прикл. мат. мех. - 1979. - 43. - С. 30-37.

18. Вельмисов П. А., Казакова Ю. А. О параметрических решениях дифференциальных уравнений с частными производными; приложения в трансзвуковой газовой динамике// Ж. Средневолж. мат. обва. - 2010. - № 4. - С. 30-36.

19. Вельмисов П. А., Молгачев А. А. Математическое моделирование в задачах динамической устойчивости вязкоупругих элементов проточных каналов. - Ульяновск: УлГТУ, 2012.

20. Вельмисов П. А., Манжсосов В. К. Математическое моделирование в задачах динамики виброударных и аэроупругих систем. - Ульяновск: УлГТУ, 2014.

21. Вельмисов П. А., Маценко П. К. О некоторых задачах внешнего пространственного обтекания тел околозвуковым потоком газа// Изв. вузов. Мат. - 1986. - № 9. - С. 10-16.

22. Вельмисов П. А., Горбоконенко В. Д, Решетников Ю. А. Математическое моделирование механической системы «трубопровод-датчик давления» // Датчики и системы. - 2003. - № 6 (49). - С. 12-15.

23. Вельмисов П. А., Решетников Ю. А. Устойчивость вязкоупругих пластин при аэрогидродинамическом воздействии. - Саратов: Изд-во Саратов. ун-та, 1994. 
24. Вельмисов П. А., Тамарова Ю. А. Асимптотические уравнения нелинейных трансзвуковых течений газа и их решения// Ж. Средневолж. мат. об-ва. - 2013. - 15, № 4. - С. 70-76.

25. Вельмисов П. А., Тамарова Ю. А. Асимптотические модельные уравнения трансзвуковых течений газа// Вестн. УлГТУ. - 2013. - № 3 (63). - С. 53-58.

26. Вельмисов П. А., Тамарова Ю. А. Математическое моделирование трансзвуковых течений// Автоматизация процессов управления - 2014. - № 1 (35). - С. 47-54.

27. Вельмисов П. А., Фалькович С. В. Неустановившиеся течения газа в соплах Лаваля с местными сверхзвуковыми зонами// Прикл. мат. мех. - 1975. - 39, № 2. - С. 271-279.

28. Вельмисов П. А., Фалькович С. В. К теории околозвуковых течений вязкого газа// Изв. вузов. Мат. - 1974. - № 5. - C. 52-61.

29. Вельмисов П. А., Решетников Ю. А., Колмановский Е. Е. Устойчивость уравнений взаимодействия вязкоупругих пластин с жидкостью// Диффер. уравн. - 1994. - 30, № 11. - С. 1966-1981.

30. Гудерлей К. Г. Теория околозвуковых течений. - М.: ИЛ, 1960.

31. Ершов Б. А., Кутеева Г. А. Колебания идеальной жидкости в прямоугольном сосуде с упругой вставкой на стенке. Учет внутреннего трения в материале вставки// Вестн. Санкт-Петерб. ун-та. - 2005. - 1, № 2. - С. 86-94.

32. Заславский Б. И., Клепикова Н. А. Об одном классе точных частных решений уравнений околозвуковых течений газа// Прикл. мат. техн. физ. - 1965. - № 6. - С. 65-68.

33. Звягин A. В. Движение вязкой жидкости в канале с упругими границами// Вестн. Моск. ун-та. Сер. 1. Мат. мех. - 2005. - № 1. - С. 50-54.

34. Казакова Ю. А. О некоторых классах решений уравнений аэрогидромеханики// Вестн. Самар. гос. техн. ун-та. Сер. физ.-мат. науки. - 2011. - № 2 (23). - С. 289-294.

35. Коллати, Л. Задачи на собственные значения. - М.: Наука, 1968.

36. Лифиии Ю. Б., Рыжов О. С. Об асимптотическом типе плоскопараллельного течения в окрестности сопла Лаваля// Докл. АН СССР. - 1964. - 154, № 2. - С. 290-293.

37. Лифшич, Ю. Б., Рыюсов О. С. О причинах образования ударных волн в соплах Лаваля// Докл. АН CCCP. - 1964. - 154, № 5. - C. 1052-1055.

38. Лифиии Ю. Б., Ръюсов О. С. О некоторых точных решениях уравнений трансзвуковых течений газа// Ж. вычисл. мат. мат. физ. - 1964. - 4, № 5. - С. 954-958.

39. Могилевич Л. И., Попова А. А. Динамика взаимодействия упругой цилиндрической оболочки с ламинарным потоком жидкости внутри нее применительно к трубопроводному транспорту// Наука и техн. транспорт. - 2007. - № 2. - С. 69-72.

40. Мокеев B. В. Конечно-элементное решение задачи гидроупругости для вязкоупругой жидкости// Пробл. машиностр. надежн. машин. - 2005. - № 2. - С. 9-86.

41. Овсянников Л. В. Исследование газовых течений с прямой дозвуковой линией// Тр. Ленинград. Военно-Воздушн. инж. акад. им. А. Ф. Можайского. - 1950. - № 33. - С. 3-24.

42. Рыжов О. С. Некоторые вырожденные околозвуковые течения// Прикл. мат. мех. - 1958. -22, № 2 . - C. 260-264.

43. Рыжов О. С. Исследование трансзвуковых течений в соплах Лаваля. - М.: ВЦ АН СССР, 1965.

44. Рыжся О. С. О работе сопел Лаваля в нерасчетных режимах// Ж. вычисл. мат. мат. физ. - 1967. 7, № 4. - C. 859-866.

45. Рыжсов О. С., Шефтер Г. М. О влиянии вязкости и теплопроводности на структуру сжимаемых течений// Прикл. мат. мех. - 1964. - 28, № 6. - С. 996-1007.

46. Соколов В. Г., Березнев А. В. Уравнения движения криволинейного участка трубопровода с потоком жидкости // Изв. вузов. Нефть и газ. - 2004. - № 6. - С. 76-80.

47. Фалькович С.В. К теории сопла Лаваля// Прикл. мат. мех. - 1946. - № 10. - С. 503-512.

48. Франкль Ф. И. Избранные труды по газовой динамике. - М.: Наука, 1973.

49. Шифрин Э. Г. Потенциальные и вихревые трансзвуковые течения идеального газа. - М.: Физматлит, 2001.

50. Adamson T. C. Unsteady transonic flows in two-dimensional channels// J. Fluid Mech. — 1972. — 52, № 3. - P. 437-449.

51. Vel'misov P. A., Tamarova Ju. A. Asymptotic research of transonic gas flows// AIP Conf. Proc. — 2017. - 1910. - 040002 . 
52. Vel'misov P. A., Todorov M. D., Kazakova Ju. A. Some classes of the solutions of aerohydromechanic equations// AIP Conf. Proc. - 2009. - 1067. - P. 427-441.

53. Karman T. The similarity law of transonic flow// J. Math. Phys. — 1947. — 26, № 3. — P. 182-190.

54. Lin C. C., Reissner E., Tsien H. S. On two-dimensional non-steady motion of a slender body in a compressible fluid// J. Math. Phys. - 1948. - 27, № 3. - P. 220-231.

55. Paidoussis $M$. P. The canonical problem of the fluid-conveying pipe and radiation of the knowledge gained to other dynamics problems across Applied Mechanics// J. Sound Vibr. — 2008. — 310, № 3. — P. 462-492.

56. Sichel M. Structure of weak non-Hygoniot shocks// Phys. Fluids. - 1963. - 6, № 5. - P. 653-662.

57. Sichel $M$. The effect of longitudinal viscosity on the flow at a nozzle throat// J. Fluid. Mech. - 1966. 25, № 4. - P. 769-786.

58. Tomotika S., Tamada K. Studies on two-dimensional transonic flows of compressible fluids// Quart. Appl. Math. - 1949. - 7, № 4. - P. 381-397.

59. Tomotika S., Hasimoto Z. On the transonic flow of a compressible fluid through an axially symmetrical nozzle// J. Math. Phys. - 1950. - 29, № 2. - P. 105-117.

Вельмисов Петр Александрович

Ульяновский государственный технический университет

E-mail: velmisov@ulstu.ru

Тамарова Юлия Александровна

Ульяновский государственный технический университет

E-mail: kazakovaua@mail.ru

Семенова Елизавета Петровна

Ульяновский государственный технический университет

E-mail: farbless@gmail.com 